\title{
Advances in The Treatment Options Towards Drug-Resistant Tuberculosis - A Review
}

Mohammed Hussen Bule ${ }^{\mathrm{a}}$, Faheem Maqbool ${ }^{\mathrm{b}}$, Ishtiaq Ahmed ${ }^{\mathrm{c}, \mathrm{d}, *}$, Kuldeep Dhama ${ }^{\mathrm{e}}$, and Hafiz

$$
\text { M. N. Iqbal }{ }^{\mathrm{f}, *}
$$

${ }^{a}$ Department of Pharmacy, Clloege of Medicine and Health Sciences, Ambo University, Ambo, Ethiopia; ${ }^{b}$ Faculty of Pharmaceutical Sciences, Government College University, Faisalabad, Pakistan; ${ }^{c}$ Department of Biochemistry, University of Agriculture Faisalabad, Pakistan; ${ }^{d}$ School of Medical Science, Gold Coast campus, Griffith University, Southport QLD 4222, Australia; ${ }^{e}$ Division of Pathology, ICAR-Indian Veterinary Research Institute, Izatnagar, Bareilly 243122, Uttar Pradesh, India; fENCIT - Science, Engineering and Technology School, Tecnologico de Monterrey, Ave. Eugenio Garza Sada 2501, Monterrey, N.L., CP 64849, Mexico; *Corresponding author Tel: +52 (81) 83582000 ext. 5561-115;

E-mail addresses: i.ahmed@griffith.edu.au, hafiz.iqbal@my.westminster.ac.uk \& hafiz.iqbal@itesm.mx

\begin{abstract}
From decades' tuberculosis (TB) affecting individual and a huge number of people die every year. It causes ill-health among millions of individuals each year and ranks as the second leading cause of death from an infectious disease worldwide. The number of TB deaths has been unacceptably increased to a large extent, and most cases are preventable if people get in time access to health care for a diagnosis and the right treatment. Multi-drug resistance is the fact that in TB patients are growing rapidly and difficult to treat. Treatment of MDR-TB is more complicated and longer than treatment of TB with no resistance. With advancements in the therapy of TB, now ideal treatments have been developed, and many combination therapies are well recommended for MDR. The development of MDR-TB can be caused by a treatment that is inadequate, given the drug susceptibility pattern of the MTB strain. Few cohort studies provided information on treatment regimens and drug resistance profiles before treatment and at failure or recurrence or genotyping information at failure or recurrence. To monitor the development of acquired drug resistance we suggest that given sufficient resources are available, TB treatment cohort studies or surveillance systems measure both the drug resistance profile and genotype information before starting treatment and at failure or recurrence. The emergence of MDR-TB and XDR-TB, the
\end{abstract}


need for new TB drug regimens and rapid DST is intuiting globally. In future, more research with a focus on MDR and TB is required to avoid and counteract its associated complications.

\section{Introduction}

Tuberculosis (TB), caused by the bacteria Mycobacterium tuberculosis, is notorious for its lethality to humans ${ }^{1,21,2}$. It causes ill-health among millions of people each year and ranks as the second leading cause of death from an infectious disease worldwide. The latest estimates included in this report are that there were 8.6 million new TB cases in 2012 and 1.3 million TB deaths ${ }^{3}$. Tuberculosis is the major infectious disease killing nearly two million people, mostly in developing countries, every year. The increasing incidence of Mycobacterium tuberculosis strains to the most cost effective anti-TB drugs is the major factor contributing to the current TB epidemic. Drug-resistant strains have involved mainly due to incomplete or improper treatment of TB patients. The resistance of Mycobacterium tuberculosis to anti-TB drugs is caused by a chromosomal mutation in encoding drug targets ${ }^{44}$.

TB organisms that are resistant to the antibiotics used in its treatment are widespread and occur in all countries surveyed. Drug resistance emerges as a result of inadequate treatment, and once TB organisms acquire resistance they can spread from person to anyone in the same way as drugsensitive TB ${ }^{55}$. The development of drug resistance by MTB is a major concern. Drug-resistant MTB strains were first recognized soon after the introduction of streptomycin in 1944, and subsequently, resistance to other TB medications was demonstrated. Spontaneous mutations that impart drug resistance to MTB occur at predictable rates ${ }^{66}$. Resistant strains are perpetuated by inappropriate treatment inadequate therapeutic regimen, poor patient compliance due to intolerable adverse drug effects, or treatment default because of inadequate support from local TB services $^{7-97-9}$.

The proportion of TB cases with drug-resistance: about 3.7\% of new tuberculosis (TB) patients in the world have multidrug-resistant strains (MDR-TB). Levels are much higher in those previously treated about 20\%. The frequency of MDR-TB varies substantially between countries. Multidrugresistant TB (MDR-TB) is caused by organisms that are resistant to the most effective anti-TB drugs (isoniazid and rifampicin). MDR-TB results from either infection with organisms which are already drug-resistant or may develop in the course of a patient's treatment. About 9\% of MDRTB cases also have resistance to two other classes of drugs, or extensively drug-resistant TB 
(XDR-TB). By March 2013, 84 countries had reported at least one XDR-TB case. Extensively drug-resistant TB (XDR-TB) is a form of TB caused by organisms that are resistant to isoniazid and rifampicin (i.e. MDR-TB) as well as any fluoroquinolone and any of the second-line anti-TB injectable drugs (amikacin, kanamycin or capreomycin) ${ }^{55}$. Even more ominous are reports of XDR-MTB strains that also demonstrate in vitro resistance to other second-line drugs, including aminoglycosides, cyclic polypeptides, fluoroquinolones, thioamides, serine analogs, and salicylic acid derivatives ${ }^{10,1110,11}$.

Treatment needs to be continued for a minimum of 20 months with four effective drugs, with the discontinuation of the injectable medication at the end of 8 months of therapy if there is a good clinical response to the intensive phase of treatment ${ }^{1212}$. If drug resistance or side effects preclude inclusion of one or more of these drugs, then, in descending order of preference, para-amino salicylate or group 5 antibiotics are recommended to provide a minimum of four effective drugs 1313. The long treatment courses are expensive and difficult to tolerate. In a population of rural Bangladeshi HIV-negative patients with pulmonary MDR-TB, without extensive additional resistance and who were not previously treated with second-line drugs, a 9-month regimen that include gatifloxacin, pyrazinamide, ethambutol, and clofazimine, and included an initial intensive 4-month phase that also included treatment with kanamycin, prothionamide, and high-dose $\mathrm{H}$, resulted in a relapse-free cure rate of almost $88 \%$ with a reasonable rate of adverse effects. Outcomes were not as good in patients treated with an ofloxacin based regimen as in the gatifloxacin-treated group ${ }^{1414}$. This study suggests that some patients with MDR-TB may be

treated successfully with a shorter regimen than currently recommended by WHO ${ }^{1215}$. Figure 1 illustrates various organs/systems effects the pulmonary tuberculosis and mechanism involved in MDR.

\section{Drug-resistant Tuberculosis}

The current MDR-TB epidemic is the result of decades of neglect for an important infectious disease, lack of resources for national TB control programs, poor case detection and inadequate/inappropriate therapy in high-burden countries. Initial outbreaks of MDR-TB in developed countries were associated with very high mortality rates both in HIV-negative and HIV- coinfected patients. Optimization of treatment regimens together with rapid diagnosis and DST for first and second-line drugs significantly improved the clinical outcome. Recent advances in the diagnosis of MDR-TB and aggressive empirical treatment of patients with several drugs in 
the initial phase of therapy have further improved the prognosis MDR-TB. However, in developing countries, the prognosis is still largely poor due to inadequate laboratory support that is critical for successful management of MDR-TB patients. Rapid and cost-effective methods for culture and DST of M. TB strains have recently been developed that are suitable for resource-poor countries. The new anti-TB drugs that are in various stages of development also offer hope that we will not soon run out of treatment options against TB and MDR-TB ${ }^{44}$. There are legitimate grounds for optimism that recent advances in TB drug development will result in achievement of some priority goals in the management of active TB. The recent licensing of aquiline and the encouraging performance of sterilizing drugs such as the 8-methoxyfluoroquinolines and nitroimidazoles in Phase IIb clinical trials are concrete signs of progress towards shorter therapy. However, this optimism should be tempered with a degree of caution. A regimen less than 6months in duration with proven efficacy against drug susceptible-TB are still awaited. Management of TB-HIV co-infection remains difficult, particularly in resource-poor settings where the disease burden is highest. The precise role of new medicinal products in the management of MDR-TB remains undefined, and Phase III clinical trials will not report for several years. Extrapolating conclusions from Phase II studies is compromised by uncertainty surrounding the validation of surrogate endpoints. Several additional questions are beyond the scope of this review; management of latent TB infection, appropriate dosing regimens for pediatric TB ${ }^{1516}$.

Overall, the last decade has been associated with the most encouraging advances in TB therapeutics since the 1960s, but these breakthroughs have still to impact on the stubbornly high global burden of TB disease. Long-term investment, political commitment, and scientific Endeavor will be crucial to ensure that progress is sustained and the benefits of recent advances reach those in the greatest need ${ }^{1617}$. With the continuing rise in the occurrence of drug-resistant strains of bacteria, new approaches to combating infections caused by these bacteria are desperately needed, particularly for Gram-negative bacteria. The one drug-one target model has limited viability, and combination therapy is the norm in the treatment of many cancers, viral infections such as HIV and tuberculosis treatment. The use of combination therapy or other drug cocktails such as antibiotic/adjuvant combinations for the treatment of other MDR bacterial infections is an attractive alternative to the development of new antibiotics, which has been demonstrated to almost invariably lead to the emergence of resistance following a short time in 
the clinic. The use of antibiotic-adjuvant combinations presents several advantages over the development of new antibiotics, namely the decreased likelihood of resistance development.

Several approaches have been discussed here, including the recent events towards traditional adjuvants such as $\beta$-lactamase inhibitors and efflux pump inhibitors, new approaches such as interference of the bacterial response to the presence of antibiotics by the targeting of bacterial signaling pathways, and the use of high throughput screening of previously approved drugs to identify compounds with unanticipated adjuvant activity. There are issues with the use of any drug combinations, most notably drug-drug interactions, and optimized drug ratios and dosing regimens to match the absorption, distribution, metabolism, and excretion properties of each compound. However, the lack of novel antibiotic classes being developed, the significant problem of resistance acquisition to therapeutic approaches that rely solely on a single bacteriostatic/bactericidal mechanism, and the clinical success of antibiotic/adjuvant combinations such as Augmentin makes the adjuvant approach an extremely attractive avenue to the development of novel therapeutics to treat multidrug-resistant bacterial infections ${ }^{1718}$. Various MDR stages along with potential therapeutic strategies are shown in Figure 2.

\subsection{Epidemiology of drug-resistant TB}

According to WHO global report 2013 shows latest estimates included in this report are that there were 8.6 million new TB cases in 2012 and 1.3 million TB deaths. Most of these TB cases and deaths occur among men, but the burden of disease among women is also high. In 2012, there were an estimated 2.9 million cases and 410000 TB deaths among women, as well as an estimated 530000 cases and 74000 deaths among children. About 75\% of these cases were in the African Region. Globally in 2012, approximately 450000 people developed MDR-TB, and there were an estimated 170000 deaths from MDR-TB. Most TB cases and deaths occur among men, but TB remains among the top three killers of women worldwide. The number of TB deaths is unacceptably large given that most are preventable if people can access health care for a diagnosis and the right treatment is provided. Short-course regimens of first-line drugs that can cure around $90 \%$ of cases have been available for decades ${ }^{33}$.

CDC report 2012 shows that a total of 9,951 new tuberculosis (TB) cases were reported in the United States, an incidence of 3.2 cases per 100,000 populations. This represents a decrease of 6.1\% from the incidence reported in 2011 and is the 20th consecutive year of declining rate. A 
total of 127 cases of multidrug-resistant TB (MDR-TB) were reported in 2011. Drugsusceptibility test results for isoniazid and rifampin were reported for $97.0 \%$ and $96.8 \%$ of culture-confirmed TB cases in 2010 and 2011, respectively. Among these cases, the percentage of MDR TB for 2011 (1.6\%) was greater than the percentage for 2010 (1.3\%). The percentage of MDR TB cases among persons without a previous history of TB was 1.3\% in 2011. For individuals with a previous history of TB, the percentage with MDR TB was 8.2\% in 2011. One case of extensively drug-resistant TB has been reported for $2012{ }^{1819}$.

According to study conducted in German 2008 In 177 (4\%) of 4,557 culture-positive TB cases, Mycobacterium tuberculosis isolates were identified as MDR-TB; an additional 7 (0.15\%) met criteria for XDR-TB ${ }^{1819}$. About 3.7\% of new tuberculosis (TB) patients in the world have multidrug-resistant strains (MDR-TB). Levels are much higher in those previously treated about 20\%. The frequency of MDR-TB varies substantially between countries. WHO estimates that there were about 0.5 million new MDR-TB cases in the world in 2011.About $60 \%$ of these cases occurred in Brazil, China, India, the Russian Federation and South Africa alone 55 .

The Africa region had approximately one-quarter of the world's cases (27\%), and the highest rates of cases and deaths about population (255 incident cases per 100000 on average, more than double the global average of 122.) The TB incidence rate at country level ranges substantially, with around 1000 or more cases per 100000 people in South Africa ${ }^{11}$. According to a study conducted in Uganda 2013 is ranked $16^{\text {th }}$ among the 22 countries with the highest TB burden, with an incidence of 299 and a mortality rate of 84 cases per 100,000 per year. MDR-TB prevalence among new and previously treated patients in Kampala is $1.1 \%$ and $11.7 \%$, respectively 1920

Ethiopia ranks $7^{\text {th }}$ in the list of the world's 22 high burden countries for TB with incidence estimated at 379/100,000 for all forms of TB and 168/100,000 for smear positive tuberculosis. The Annual Risk of TB Infection is estimated at 2.2\%. Tuberculosis (TB) is one of the leading infectious diseases in Ethiopia. According to the national routine report and the global TB report 2012, more than 155,000 new TB cases of all forms are annually notified. Drug-resistant TB has also become a major challenge of the TB control program in Ethiopia. According to the first national drug resistance survey completed in 2005, the rate of MDR-TB is 1.6 and $11.8 \%$ among new and previously treated cases, respectively ${ }^{2021}$. 
According to the WHO global report 2013 estimates, the incidences of TB of all forms stand at 247 per 100,000 populations, respectively. The prevalence and mortality of Tuberculosis of all forms are estimated to be 224 and 18 per 100,000 populations respectively. In the year 2012 Ethiopia registered 147,592 cases of TB. According to latest estimates, Ethiopia stands $9^{\text {th }}$ in the list of High Burden Countries for TB. It is estimated that MDR-TB represents about $16 \%$ of new TB cases and 12 of re-treatment cases ${ }^{33}$.

\subsection{Treatment of drug resistant $T B$}

Treatment of MDR-TB is more complicated and longer than treatment of TB with no resistance. The main indications for TB culture and DST in search for MDR-TB are: Retreated cases who fail to respond to category II regimen, Chronic cases, new cases that contact cases of known or suspected MDR cases and unclear treatment failure with good compliance. The prevention of MDR can only be achieved through proper TB case detection, rational diagnosis, standard treatment and, most of all, successful follow-up and high adherence of patients. Clearly, an effective DOTs program is the best weapon against MDR. There are not enough second line options to cope with the widespread surge of MDR and XDR TB for established and proven MDR, anti-TB drugs efficient for MDR (Second-line TB drugs) will gradually be accessible in Ethiopia. It is however of crucial importance that this "last chance" second line drugs are used in a very rational way to maintain their effectiveness. If they are not properly used, resistance will extend to these second-line drugs. Every health worker has to be aware that, beyond these secondline TB drugs, there is no another option left, and the presence of untreatable communicable TB in the community would be a serious threat for all, especially health workers. Recently much occupational and working conditions have been assumed to be a major cause to initiate many diseases. Like dental amalgam caused many problems due to mercury toxicity ${ }^{2122}$. Such complications need to be addressed on time. The treatment for MDR is complex, very long and expensive; severe side effects are quite common, and the success rate is lower than with common TB (non-MDR). MDR-TB patients should be treated with a combination of second line drugs and, if advisable, fist line TB drugs that still have proven efficacy. Regimens should consist of drugs with either certain or almost certain, effectiveness. The treatment should be standardized or individually adjusted by result of Drug Sensitivity Testing. Since susceptibility to all TB drugs cannot be assessed routinely, and as a comprehensive result may not be available for several weeks, five or six drugs are recommended initially. The first phase, which injectable drug, should 
be a minimum of 6 months and initial treatment may be extended if the patient does not convert both smear and culture. The entire treatment period is 18-24 months after smear and culture conversion ${ }^{2223}$. The names and structures of various antituberculosis drugs are summarized in Table 1.

The development of MDR-TB can be caused by a treatment that is inadequate, given the drug susceptibility pattern of the MTB strain. The information can be used to advocate the need for adequate treatment of patients, based on drug resistance profiles. Few cohort studies provided information on treatment regimens and drug resistance profiles before treatment and at failure or recurrence or genotyping information at failure or recurrence. To monitor the development of acquired drug resistance we suggest that given sufficient resources are available, TB treatment cohort studies or surveillance systems measure both the drug resistance profile and genotype information before starting treatment and at failure or recurrence. This would enable future larger studies to assess the risk of resistance development due to inappropriate treatment ${ }^{99}$. With the continuing rise in the occurrence of drug-resistant strains of bacteria, new approaches to combating infections caused by these bacteria are desperately needed, particularly for Gramnegative bacteria. The one drug-one target model has limited viability, and combination therapy is the norm in the treatment of many cancers, viral infections such as HIV and tuberculosis treatment. The use of combination therapy or other drugs such as antibiotic/adjuvant combinations for the treatment of other MDR bacterial infections is an attractive alternative to the development of new antibiotics, which has been demonstrated to almost invariably lead to the emergence of resistance following a short time in the clinic. The use of antibiotic-adjuvant combinations presents several advantages over the development of new antibiotics, namely the decreased likelihood of resistance development. Several approaches have been discussed here, including the recent events towards traditional adjuvants such as $\beta$-lactamase inhibitors and efflux pump inhibitors, new approaches such as interference of the bacterial response to the presence of antibiotics by the targeting of bacterial signaling pathways, and the use of high throughput screening of previously approved drugs to identify compounds with unanticipated adjuvant activity. There are issues with the utilization of any drug combinations, most notably drug-drug interactions and optimized drug ratios and dosing regimens to match the absorption, distribution, metabolism, and excretion properties of each compound. However, the lack of novel antibiotic classes being developed, the significant problem of resistance acquisition to therapeutic 
approaches that rely solely on a single bacteriostatic/ bactericidal mechanism, and the clinical success of antibiotic/adjuvant combinations such as Augmentin makes the adjuvant approach an extremely attractive avenue to the development of novel therapeutics to treat multidrug-resistant bacterial infections ${ }^{17} 18$.

The current MDR-TB epidemic is the result of decades of neglect for an important infectious disease, lack of resources for national TB control programs, poor case detection and inadequate/inappropriate therapy in high-burden countries. Initial outbreaks of MDR-TB in developed countries were associated with very high mortality rates both in HIV-negative and HIV-co-infected patients. Optimization of treatment regimens together with rapid diagnosis and DST for first- and second-line drugs, significantly improved the clinical outcome. Recent advances in the diagnosis of MDR-TB and aggressive empirical treatment of patients with several drugs in the initial phase of therapy have further improved the prognosis MDR-TB. However, in developing countries, the prognosis is still largely poor due to inadequate laboratory support that is critical for successful management of MDR-TB patients. Rapid and cost-effective methods for culture and DST of MTB strains have recently been developed that are suitable for resource-poor countries. The new anti-TB drugs that are in various stages of development also offer hope that we will not soon run out of treatment options against TB and MDR-TB ${ }^{44}$.

Combinations of first-line and second-line drugs are useful for the treatment of multidrugresistant and extensively drug-resistant tuberculosis according to results of drug susceptibility testing. Second-line drugs include aminoglycosides (kanamycin and amikacin), cycloserine, terizidone, ethionamide, prothionamide, capreomycin, aminosalicylic acid, and fluoroquinolones (including ofloxacin, levofloxacin, gatifloxacin, and moxifloxacin). The percentage of patients with multidrug-resistant tuberculosis who are cured is estimated to be no more than $69 \%$, even when treated for more than 18 months with directly observed treatment ${ }^{23,2424,43}$.

Updated guidelines now recommend that 4 or more drugs with limited or highly probable effectiveness against TB should be included in treatment regimens. The selection of TB drugs is structured such that treatment options proceed from group 1 through to group 5. Among the group 1 drugs, pyrazinamide or ethambutol can be selected for MDR-TB cases. However, the World Health Organization (WHO) does not recommend ethambutol, citing its limited efficacy against MDR-TB. The effectiveness of rifabutin for MDR-TB is unclear, and this agent is thus not routinely included in MDR-TB regimens. Having selected the group 1 drugs, one injectable group 
2 drug should then be chosen as part of the regimen. The concomitant use of 2 or more injectable drugs is not recommended. Also, the WHO does not recommend streptomycin for MDR-TB because streptomycin-resistance rates are high, as is the frequency of ototoxicity, compared with other injectable drugs. Capreomycin is not available in Korea and can only be purchased from the Korea Orphan Drug Center. Capreomycin is also very expensive. Kanamycin and amikacin have nearly $100 \%$ cross-resistance. Amikacin is usually injected intravenously (even though intramuscular injection is possible), and therefore kanamycin is the drug of choice for MDR-TB in Korea. The WHO recommends kanamycin as the first injectable drug for MDR-TB. The concomitant use of 2 or more fluoroquinolones is not recommended. At this stage in the regimen design, only 3 or fewer agents have been selected (pyrazinamide, one injectable drug or one fluoroquinolone). Hence, group 4 drugs should also be included in the regimen. In Korea, the open group 4 drugs include prothionamide, cycloserine, and para-aminosalicylic acid. These can be selected by drug susceptibility results, previous treatment history, and the resistance pattern in the region. Prothionamide is the preferred choice, followed by cycloserine then paraaminosalicylic acid. The WHO has recommended an empirical regimen for MDR-TB comprising pyrazinamide, one injectable agent (kanamycin), one fluoroquinolone (moxifloxacin or levofloxacin), prothionamide, and either cycloserine or para-aminosalicylic acid (if cycloserine cannot be used). Ethambutol may be utilized but is not included in the count of effective drugs. Traditionally the recommended treatment duration for MDR-TB has been 18 to 24 months, including a period of 12-18 months after culture conversion. According to a recent meta-analysis of 9,153 MDR-TB patients, an intensive phase of at least eight months and a total duration of at least 20 months are recommended in patients without any previous MDR-TB treatment ${ }^{1313}$.

DOTs are a proven cost-effective TB treatment strategy. A combination of technical and managerial components, DOTs quickly makes infectious cases non-infectious and breaks the cycle of transmission. Using DOTs also prevents the development of drug-resistant strains of TB that are often fatal and very expensive to cure. Multi-drug-resistant TB is both an individual tragedy and a reflection of poor program performance. The top priority is to prevent the emergence of MDR-TB by ensuring a low default rate of cases treated with first-line anti-TB drugs. If MDR-TB has emerged in a particular area, it should be treated in addition to improving the core treatment. In this situation, accurate and reliable drug susceptibility testing, methods to 
support patients to ensure direct observation of complete treatment, and the use of maximally effective regimens must be ensured. Patients with MDR-TB have a good chance for a cure with second-line drugs, hence the treatment, if it is to be provided, should be optimally selected and administered. Second-line drugs should not be kept in reserve, and the treatment observation must be ensured ${ }^{66}$.

Extensively drug-resistant tuberculosis (XDR-TB) is a type of multidrug-resistant tuberculosis (MDR-TB) that is resistant to two of the first-line drugs isoniazid and rifampicin as well as to the second-line medications that include a fluoroquinolone such as ciprofloxacin and at least one of the injectable drugs which may be an aminoglycoside such as amikacin or kanamycin, or a polypeptide-like capreomycin, or a thioamides such as ethionamide, or cycloserine or paminosalicylic acid. Because the treatment regimen for TB is long and complex, many patients are unable to complete the course of therapy, enabling their disease to develop drug-resistance. Once a drug-resistant strain has developed, it can be transmitted directly to others. XDR TB being resistant to the front-line drugs and two or more of the six classes of second-line drugs, this makes it virtually untreatable and infected people are particularly at a greater risk.

Therefore, XDR TB could have a bigger impact on developing nations because there is a high prevalence of HIV and lack of capacity to quickly and efficiently diagnose and identify the disease. To prevent XDR-TB, there is an urgent need for new diagnostic tools and new and more efficient anti-TB drugs and vaccines to be developed ${ }^{2525}$. XDR-TB strains have arisen due to the mismanagement of individuals with MDR-TB. The global epidemic of drug-resistant tuberculosis is due to a combination of acquired resistance and initial transmission. Because XDR-TB is resistant to the most powerful first-line and second-line drugs, patients are left with treatment options that are much less efficient and often have worse treatment outcomes ${ }^{2626}$.

The emergence of XDR-TB raises concerns about the possibility of epidemics of virtually untreatable TB. Such epidemics could result in excessive mortality and substantial financial and infrastructure burden for public health and TB control programs. XDR TB is much more expensive to treat, with hospitalization costs. A major outbreak of XDR TB in the United States would constitute a substantial drain on public health resources and could quickly deplete the existing state and local TB budgets and have an adverse impact on progress toward TB elimination. Treatment failures and subsequent death are more common among patients with XDR-TB, and the drugs available to treat XDR TB are associated with serious adverse effects. 
Because persons who are infected with HIV or who have other immune-compromising conditions (e.g., diabetes) are more vulnerable to progressing to active TB disease, infection with XDR TB is of particular concern among these persons. In countries with high rates of HIV and limited healthcare resources, substantial numbers of XDR TB cases are likely to present ${ }^{2727}$.

Culture conversion guides the recommended total duration of treatment. Despite emerging evidence that shorter regimens may be efficacious, WHO guidelines recommend continuing therapy for a minimum of 18 months after culture conversion. Extension of treatment of 24 months may be indicated in chronic cases with extensive pulmonary damage. The length of treatment for XDR tuberculosis has not been established and is often based on individual clinical presentations.

Good outcome is seen with treatment of at least 18 months for oral agents, and of at least 8 months after culture conversion for injectable drugs in most cases the regimen does not contain 5 effective drugs, and clinician may have to adopt reinforcing strategies like extending the duration of treatment with the injectable agent or extending the duration of the whole regimen with the addition of other drugs which have questionable activity against MDR-TB ${ }^{2828}$.

Standardized treatment regimen category IV Regiment, comprising of 6 drugs (kanamycin, levofloxacin, ethionamide, cycloserine, pyrazinamide, and ethambutol) during 6-9 months of the intensive phase and four drugs (levofloxacin, ethionamide, cycloserine, and ethambutol) during the 18 months of the continuation phase. P-aminosalicylic acid is included in the regimen as a substitute drug if any bactericidal drug (kanamycin, levofloxacin, pyrazinamide, or ethionamide) or the two bacteriostatic (ethambutol and cycloserine) drugs are not tolerated. This Catagory IV regimen is highly suitable for high TB prevalent nations as well as low to middle-income countries. An injectable agent should be given for at least six months, and the whole treatment duration is minimum 18 months beyond sputum conversion. WHO advises that injectable drugs in the treatment of XDR-TB to be continued for the extended duration of 12 months or possibly the whole treatment ${ }^{1329}$. Currently, the most potent drug in group 5 is linezolid, and it should thus always be included in XDR-TB. The Recent prospective trial has shown that Linezolid is effective for achieving culture conversion among patients with treatment-refractory XDR-TB. Linezolid added to the regimen of patients failing standard XDR-TB treatment has been demonstrated to improve culture conversion, but longer-term outcomes are unknown, and cost and toxicity are major concerns ${ }^{29,3030,31}$. 


\subsection{Mechanism of resistance}

A drug resistance mechanism in MTB is crucial for the development of rapid methods for drug resistance detection and new anti-TB drugs to treat MDR-TB/XDR-TB patients ${ }^{4}$. The everincreasing drug use in response to growing TB incidences has resulted in a steady evolution of $\mathrm{M}$. tuberculosis strains that are progressively resistant to the available drugs. Besides the matter of selective survival, recent studies show that exposure of bacterial cells to sub-lethal levels of bactericidal antibiotics promotes cellular mutagenesis leading to increased mutations in other drug resistance genes ${ }^{3132}$. The first-line drugs, INH and RIF, are the backbone of treatment for TB. If resistance develops to either of the two drugs, the patients can still be successfully managed with the available drugs, although treatment duration is increased, particularly for infections caused by RIF-resistant strains. Among clinical M. Tuberculosisisolates, mono-resistance to INH is common while mono-resistance to RIF infrequently occurs ${ }^{44}$.

Early molecular studies identified katG (encoding catalase-peroxidase) and rpoB (encoding bsubunit of RNA polymerase) genes as major targets conferring resistance of MTB to INH and RIF, respectively $4,64,6$. Resistance to INH occurs more frequently than for most anti-TB drugs, at a frequency of 1 in $10^{6}$ bacilli in vitro ${ }^{3233}$. Mechanism of resistance to isoniazid is the occurrence of mutations in its activator, KatG, whereas mutations in the inhA gene represent the second most common mechanism ${ }^{3334}$. Mutations in the promoter region of inhA, causing overexpression ofInhA, or by mutations at the InhA active site, lowering InhA affinity for INH.KatG mutation can be associated with inhA mutations, leading to higher levels of INH resistance ${ }^{3233}$. KatG, a catalase-peroxidase involved in the degradation of harmful reactive oxygen intermediates, is required to convert the prodrug isoniazid into its bioactive form. While the most frequently detected resistance mutations in katG at amino acid position 315 only confer a low fitness cost and do not abolish the function of KatG other, more deleterious mutations in this gene were shown to be associated with compensatory mutations in the regulatory region of ahpc. The resulting over-expression of the ahpC gene product, another peroxidase, is believed to partly compensate for the loss of KatG activity ${ }^{3435}$. Resistance to RMP occurs at a frequency of $10^{-7}$ to $10^{-8}$ as a result of mutations in the beta subunit of RNA polymerase encoded by the gene rpoB. Mutations at positions 531, 526 and 516 in rpoB are among the most frequent (96\%) in RMPresistant strains ${ }^{3131}$. M. tuberculosis strains resistant to rifampicin are also resistant to isoniazid 3334. 
Mutations are situated within rpoAandrpo $\mathrm{C}$, encoding $\alpha$ - and $\beta$ '-subunits of the RNA polymerase, respectively, and alleviate the fitness cost associated with resistance mutations in $\mathrm{rpoB}$, the $\beta$ subunit of the RNA polymerase and target of Rifampicin. Drug-resistant TB can be “acquired” de novo during treatment, or it can occur through the transmission of already resistant strains; the latter being referred to as "primary resistance.” Primary resistance poses a particular challenge as less than $20 \%$ of the estimated MDR-TB cases in the world are believed to be properly diagnosed, mainly due to the lack of appropriate laboratory infrastructure in low-income areas that bear the highest disease burden ${ }^{3435}$. Drug-resistant TB has microbial, clinical, and programmatic causes. From a microbiological perspective, the resistance is caused by a genetic mutation that makes a drug ineffective against the mutant bacilli. An inadequate or poorly administered treatment regimen allows drug-resistant mutants to become the dominant strain in a patient infected with $\mathrm{TB}^{3536}$.

\subsection{Drug-resistant TB prevention and control}

The overarching goal of the Stop TB Strategy is to achieve 2015 global targets for reductions in the burden of disease caused by TB. The target set within the United Nations Millennium Development Goals (MDGs) is that TB incidence should be falling by 2015. Besides incidence, four other TB indicators are included in the MDG monitoring framework: the prevalence rate, the mortality rate, the case detection rate (the number of notified cases divided by the estimated number of incident cases in the same year, expressed as a percentage), and the treatment success rate (the percentage of TB patients who are successfully treated). The Stop TB Partnership adopted the MDG target and also set global targets to halve TB prevalence and death rates by 2015 compared with their levels in 1990. The scale at which interventions included in the Stop TB Strategy need to be implemented to achieve the 2015 targets for reductions in disease burden, and the associated funding requirements, have been described in Global Plans developed by the Stop TB Partnership ${ }^{3637}$.

Tuberculosis prevention and control has been established from the time where TB has identified as one of the major public health problems in Ethiopia for about five decades. The effort to control tuberculosis began in the early 1960s with the establishment of TB centers and sanatoriums in three major urban areas in the country. These centers practically had not a significant impact on reducing the burden of the disease in the country. The Central Office of the National Tuberculosis Control Programme was established in 1976. In 1992 a standardized and 
well-organized TB program, incorporating directly observed short-course treatment, was implemented in a few pilot areas of the country. At present, tuberculosis control strategy in Ethiopia relies on WHO recommended Stop TB Strategy, and it has been implemented in the country since $2006^{2223}$.

The Federal Ministry of Health of Ethiopia has taken a number of commendable initiatives in addressing major public health problems including drug susceptible and resistant TB in the country, that improving access to the neediest through the deployment of Health Extension Workers, construction of additional health facilities, building a nationwide diagnostic capacity for drug-resistant TB, and successfully conducted the first population-based TB prevalence survey in 2011. The results of the study have been instrumental in providing a robust estimate of the TB burden against the previous estimates which had overestimated the actual magnitude of the disease and it has been shown that AFB smear microscopy alone detected only a small proportion of TB cases, while chest X-rays proved a sensitive tool for TB screening. Based on evidence from around the world and the lessons from the National TB Prevalence Survey and other operational research projects of limited scope, it could be argued that the investigation has an important place and can play a critical role in providing the evidence base for all the stages of program planning, implementation and monitoring and evaluation. Operational research is fundamental to maximize the advances already achieved in TB control through the strengthening of programs and health services, and alleviation of risks and determinants ${ }^{3738}$.

In February 2009, the MDR-TB treatment program in Ethiopia was initiated as a pilot project at St. Peter Hospital in the capital city. The treatment comprised of a standard regimen of kanamycin, levofloxacin, cycloserine, ethionamide and Para-aminosalicylic acid. Following the successful pilot in 2009, the treatment program expanded to the northern part of Ethiopia at Gondar University Hospital in November 2010. Later, a third treatment center was opened at the ALERT hospital also located in Addis Ababa in 2011. These sites were all renovated with the support of several partners to make them suitable for MDR-TB treatment. The expansion of treatment sites reduced the waiting list for over 250 patients in 2009 to less than 100 at the beginning of 2012. The Ethiopia treatment policy requires admission of patients during the intensive phase for 4-8 weeks, to one of the three MDR hospitals. Patients are only discharged after sputum smear conversion. During the continuation phase, patients get their treatment from selected peripheral health facilities and take treatment under the supervision of a treatment 
supporter. Despite all efforts to rapidly set up the services and increase access to diagnosis and treatment, there is still not enough admission capacity to take up the WHO estimated numbers of MDR-TB patients. To accelerate scale-up of MDR treatment, the FMOH considers the introduction of the ambulatory model of care. Sites that will be selected for ambulatory care are those with minimum TB control requirements in place, which include well ventilated TB clinics, admission capacity for a small number of patients in case of side effects, staff trained on MDRTB and TB IC as well as equipped with personal protective materials ${ }^{3839}$.

\subsection{The need to advance drugs against drug-resistant TB}

All four of the most effective first-line oral medicines (rifampicin, isoniazid, pyrazinamide, and ethambutol) must be taken together during the first two months of treatment, and two (rifampicin and isoniazid) for a subsequent four months in the continuation phase, causing issues with patient adherence. When given in sub-optimum programmatic conditions, these regimens are associated with high rates of non-adherence and increased mortality and can create chronic cases of infectious drug-resistant tuberculosis. Thus, a priority for drug development has been for new tuberculosis drugs that will shorten regimens. Potent sterilizing medications that can shorten treatment duration to 2 months or less would improve adherence, and reduce program supervision and distribution costs. Furthermore, drugs that would reduce both total lengths of therapy and frequency of drug intake would be ideal ${ }^{4,394,40}$.

The new drugs are needed to tackle the growing global problem of multidrug-resistant and extensively drug-resistant tuberculosis. Patients with multidrug-resistant tuberculosis need a combination of second-line and third-line anti-tuberculosis drugs, which are much more expensive, more toxic, and less effective than are standard treatments ${ }^{11}$. WHO recommendations for the treatment of multidrug-resistant or extensively drug-resistant tuberculosis include secondline drugs and treatment duration of 18-24 months or longer ${ }^{1515}$. These guidelines are based on low-grade evidence, expert opinion, and little observational data, and do not have proof based on data from randomized controlled trials. Implementation of these guidelines results in a wide range of treatment regimens that are dependent on the availability of drug-susceptibility testing cost physician preference, and drug availability in developing countries. Thus, new regimens for multidrug-resistant or extensively drug-resistant tuberculosis that are shorter, more tolerable, and more efficient, and have been trialed under programmatic conditions, are urgently needed ${ }^{44}$. To abolish obstacles to effective treatment of TB in HIV-positive individuals and new therapies are 
needed for improved treatment of persons with latent tuberculosis infection from both drugsensitive and drug-resistant strains of MTB before it converts into active disease ${ }^{39,4040,41}$.

\subsection{Fluoroquinolone}

Fluoroquinolones are broad-spectrum antimicrobial drugs that target DNA gyrase. Several members of this class have been used as second-line drugs for the treatment of MDR-TB ${ }^{2443}$. Due to their excellent oral bioavailability, bactericidal activity against M. tuberculosis, lack of crossresistance with existing anti-TB drugs and favorable safety and tolerability profile, they have been employed off-label against MDR-TB and are now regarded as cornerstone agents for this disease $^{88}$.

\section{Moxifloxacin}

Study Investigated from John Hopkins University replacement of ethambutol or isoniazid with moxifloxacin or gatifloxacin in the first-line regimen has the potential to improve treatment. Replacement of ethambutol with moxifloxacin or gatifloxacin, each at $400 \mathrm{mg}$, is further supported by the results of three recent Phase II trials. They described greater culture conversion with moxifloxacin at 8 weeks and shorter time to culture conversion with moxifloxacin during the first 2 months 16,2417, 43. The technique of examining EBA has also been used to study moxifloxacin. Two moxifloxacin EBA studies have demonstrated that moxifloxacin has EBA superior to that of rifampin and perhaps comparable to that of isoniazid ${ }^{4144}$. A chemical structure of moxifloxacin is shown in Figure 3.

\section{Gatifloxacin}

The OFLOTUB trial shows two phase 3 trials of shorter duration first-line tuberculosis treatment have now completed patient enrolment and treatment and replaced Ethambutol with gatifloxacin in a four-month regimen ${ }^{4242}$. Newer generation fluoroquinolones significantly improve cure or treatment completion among XDR-TB patients. Among predominantly second-line treatmentnaïve MDR-TB patients, high relapse-free cure rates with good tolerance have been achieved by the inclusion of gatifloxacin $400-800 \mathrm{mg}$ once daily for at least nine months ${ }^{4345}$. A chemical structure of gatifloxacin is shown in Figure 4.

\subsection{Oxazolidinone}


The oxazolidinones exert their anti-TB activity by inhibiting protein synthesis through a novel mechanism by blocking the formation of the ribosomal initiation complex ${ }^{8,168,17}$.

\section{Linezolid}

Linezolid is the first oxazolidinone antimicrobial agent ${ }^{24,4443,46}$ that demonstrated in vitro activity against both drug-susceptible and drug-resistant isolates of MTB without showing crossresistance with the standard anti -Tuberculosis agents ${ }^{2443}$. A chemical structure of linezolid is shown in Figure 5. High maximal serum concentration, MIC $_{90}$ for Mycobacterium tuberculosis (0.5-1 mg/L) and excellent penetration into bronchial mucosa and MIC of LZD along with the slow growth of Mycobacterium tuberculosis contribute to effective daily-half dosage ${ }^{4446}$. The only currently marketed oxazolidinone, linezolid, has a MIC range of $0.125-1 \mathrm{mg} / \mathrm{mL}$, with an $\mathrm{MIC}_{50}$ of $0.5 \mathrm{mg} / \mathrm{mL}$ and an $\mathrm{MIC}_{90}$ of $1 \mathrm{mg} / \mathrm{ml}$. As a result, it has been used outside of labeled indications in difficult-to-treat cases of MDR- and XDR-TB ${ }^{88}$.

\section{PNU 100480 (Sutezolid)}

It is classified in oxazolidinones with current investigations in Phase I and more potent activity against Mycobacterium tuberculosis than LZD which is the only currently marketed oxazolidinone ${ }^{4446}$. A chemical structure of PNU 100480 is shown in Figure 6. The anti-TB activity of PNU-100480 was first reported in1996. Recent experiments in the murine model show it to be more active than linezolid even when serum concentrations are considerably lower. A long-term experiment demonstrated that the addition of PNU-100480 at $160 \mathrm{mg} / \mathrm{kg}$ shortened the duration of treatment necessary to prevent relapse, suggesting that PNU-100480 has sterilizing activity that could improve the treatment of drug-susceptible as well as drug-resistant $\mathrm{TB}^{88}$.

\subsection{Diarylquinoline}

\section{TMC-207(Bedaquiline)}

TMC207 (formerly R207910) is a diarylquinoline compound with a new mechanism of action by inhibiting mycobacterial adenosine triphosphate synthase ${ }^{44,4546,47}$. A chemical structure of TMC207 is shown in Figure 7. The target of TMC207 relied on sequence analysis of a single mutant of MTB, and two mutants of M. smegma is that were resistant to the drug and binding of TMC207 to the oligomeric and proteolipid subunit c of mycobacterial ATP synthase leads to 
inhibition of ATP synthesis, which subsequently results in bacterial death ${ }^{4345}$. TMC207 is highly potent against both drug-susceptible and drug-resistant strains of MTB (first and second line

drugs) ${ }^{4648}$. Minimal inhibitory concentration (MIC) ranging from 0.002 to $0.06 \mu \mathrm{g} / \mathrm{ml}$ and with a $\mathrm{MIC}_{50}$ of $0.03 \mu \mathrm{g} / \mathrm{m}{ }^{4547}$. The preclinical trial of the early bactericidal and sterilizing activity of TMC207 has been obtained in the mouse model. The daily administration of TMC207 (25 mg/kg) to experimentally infected mice reduced the bacillary load by approximately $2.5-3.0 \log _{10}$ per month. The drug, administered alone, was as active as the standard daily regimen of rifampicin plus isoniazid plus pyrazinamide; when substituting each first-line drug with TMC207, the activity of each combination containing TMC207 was significantly improved about that of the standard regimen ${ }^{4547}$. Ability to rapidly decrease bacillary load (EBA) of aquiline was assessed in newly diagnosed sputum positive patients. A total of 75 patients were randomized, they were given once daily either aquiline (25,100 or $400 \mathrm{mg}$ ) or $600 \mathrm{mg}$ rifampicin or $300 \mathrm{mg}$ isoniazid for seven days ${ }^{4749}$. TMC-207 at a dose of $400 \mathrm{mg}$ daily demonstrated late (5-7 days) bactericidal activity similar to that of isoniazid or rifampin ${ }^{3940}$. According to a study conducted by Tianyu Zhang et al. in 2011 shows TMC207 has strong sterilizing activity and may enable treatment of DR-LTBI in 3-4 months ${ }^{4648}$.

\subsection{Nitroimidazole}

The anti-TB effect of this class was identified in the 1990s and is principally due to inhibition of mycolic acid synthesis in the mycobacterial cell wall ${ }^{1617}$. Currently, two nitroimidazoles are in clinical development. These are the nitroimidazole-oxazine PA824, which is being developed by the TB Alliance, and the dihydroimidazole-oxazole OPC67683, which is being developed by Otsuka Pharmaceutical ${ }^{4144}$.

\section{OPC-67683 (Delamanid)}

OPC-67683 is a nitroimidazole-oxazole developed after members of the class were identified in a screen for mycolic acid synthesis inhibitors. Like the related nitroimidazole-oxazine, PA-824, it appears to undergo reductive nitro activation and inhibits ketomycolic acid synthesis as at least one of its mechanisms of action ${ }^{88}$. A chemical structure of OPC-67683 is shown in Figure 8. OPC-67683 is a nitroimidazole-oxazole with cross-resistance to PA-824 without cross-resistance to current anti-TB drugs. It is more potent than PA-824 in vitro (4-16 times) and in vivo with a MIC range of $0.006-0.024 \mathrm{~g} / \mathrm{mL}$ and minimal bactericidal dose which resulted in a 2 
$\log _{10}$ reduction in CFU of $2.5 \mathrm{mg} / \mathrm{kg}$ in mice, compared with $50 \mathrm{mg} / \mathrm{kg}$ for PA-824 in a similar model ${ }^{4446}$. A Phase III study of demand in MDR-TB is now underway with primary end-points based on time to sputum culture conversion in the first six months and secondary endpoints based on the durability of culture conversion out to 30 months ${ }^{1617}$.

\section{PA-824}

PA-824 is a nitroimidazole that has sterilizing activity against drug-susceptible and drug resistant $\mathrm{TB}$ and both active and dormant organisms induced in the hypoxic condition in vitro. The MIC of PA-824 is $0.015-0.25 \mathrm{mg} / \mathrm{ml}^{44} 46$. A chemical structure of PA-824 is shown in Figure 9. PA-824 has now entered phase 2 trials, the advantage that patients with multidrug-resistant tuberculosis can be included into the same recruitment process as those with treatment-sensitive tuberculosis 4050 .

PA-824 demonstrates dose-dependent bactericidal and sterilizing activity in a murine model of TB. At a daily dose of $100 \mathrm{mg} / \mathrm{kg}$ in mice, it has a bactericidal activity which approaches that of isoniazid and sterilizing activity comparable to that of rifampicin. The combination of PA-824 with rifampicin and pyrazinamide results in more rapid lung culture conversion than the standard first-line regimen of isoniazid, rifampicin, and pyrazinamide ${ }^{88}$. In South Africa, a partially blinded Phase IIa clinical study demonstrated that oral PA-824 monotherapy at 200, 600, 1000 or $1200 \mathrm{mg}$ caused an average drop of $0.1 \log _{10} \mathrm{CFU} / \mathrm{ml}$ sputum during the first two weeks of DS-TB treatment, only slightly below that of RHZE ${ }^{1617}$.

\subsection{Pyrrole(LL3858)}

LL-3858 was discovered in 2004 with reporting of potential killing of both drug susceptible and drug- resistant Mycobacterium tuberculosis bacilli in vitro and in vivo (murine/mice model) via unknown target and mechanism of action ${ }^{46}$. Additive activity in combination with first-line drugs

in the murine model was also described ${ }^{88}$. A chemical structure of Pyrrole (LL3858) is shown in Figure 10.

\subsection{Diamine (SQ109)}

SQ109 is a 1, 2-ethylenediamine that targets MmpL3 in MTB. MmpL3 is a mycolic acid transporter required for incorporation of mycolic acid into the MTB cell wall. SQ109 is in Phase II clinical trials for DS-TB. A chemical structure of SQ109 is shown in Figure 11. SQ109 had 
both the best antibacterial activity and the most promising toxicity, MTD and PK parameters of all of the active drug compounds ${ }^{4851}$. SQ109 is an ethylenediamine identified by screening a library of ethambutol derivatives. It has a MIC of $0.16-0.64 \mathrm{mg} / \mathrm{mL}$, which does not appear to be affected by resistance to ethambutol. The mechanism of action of SQ109 remains to be fully elucidated. The induction of Rv0341 implicates inhibition of cell wall synthesis but, in addition to the lack of evidence of cross-resistance, gene expression analysis suggests it may not be the same as that of ethambutol ${ }^{8,168,17}$. It kills MTB inside macrophages at a level equivalent to INH and superior to EMB. At its MIC, it reduces intracellular MTB by 99\%. Intracellular activity is important because MTB is facultative intracellular pathogens: they are phagocytosed by pulmonary macrophages but halt the maturation of the phagosome to create a protected cellular niche for their replication ${ }^{4851}$.

\subsection{Capuramycins (SQ641)}

SQ641, a semisynthetic antibiotic, is the lead drug candidate selected from a 7000-compound library of semisynthetic nucleoside-based translocase 1 (TL-1) inhibitors developed as potential treatments for bacterial infections. It is particularly active against MTB causing tuberculosis, nontuberculosis mycobacterial pneumonia, and pneumonia caused by Streptococcus pneumonia, Crohn's disease, and Staphylococcus auras, including the methicillin-resistant strain that causes skin and soft tissue infections. The capuramycin class of antibacterial antibiotics inhibits the TL-1 enzyme which is essential for the construction of the cell wall and is present only in bacteria. Because the TL-1 enzyme is absent in eukaryotic cells, it is an attractive target for antibiotic development. Despite the ubiquity of TL-1 in bacteria, capuramycin and its analogs, including SQ641, are remarkably specific for Mycobacteria and certain Gram-positive bacteria, making it a promising candidate for a future regime ${ }^{43}$.

\subsection{Benzothiozinones (BZT043)}

A new class of anti-TB agent is called benzothiazinones. It inhibits the major target enzyme, decaprenylphosphoryl-ß-D-ribose-2-epimerase and contributes to the cessation of deca-prenyl phosphoryl arabinose formation which is required for the synthesis of the cell wall arabinans of MTB. A chemical structure of benzothiazinones is shown in Figure 12. PBTZ-169 and BTZ-043 are in late-stage clinical development. Both drugs use a novel mechanism of action that inhibits the enzyme decaprenylphosphoryl- $\beta$-D-ribose 2'epimerase (DprE1) in MTB. Inhibition of this 
enzyme prevents the formation of decaprenyl phosphoryl arabinose. Both compounds show a 100-1000-fold reduction of colony-forming units in a chronic mouse model. By targeting the mycobacterial cell wall, benzothiazinones affect replicating bacilli more than dormant bacilli ${ }^{4050}$.

\subsection{Imidazopyridine}

Q203 is an optimized compound made from imidazopyridines, a new class of drugs that inhibit mycobacterial growth by blocking the respiratory cytochrome bc1 complex essential to maintain the proton gradient and ATP synthesis. Although the drug has a similar target as aquiline, it inhibits ATP synthesis more potently in both aerobic and hypoxic conditions. It is active against multidrug-resistant and extensively drug-resistant isolates of $\mathrm{M}$. Tuberculosis from human beings, and data from mice models show a 100-1000-fold reduction of colony-forming units and a blocking of granuloma formation ${ }^{4050}$.

\subsection{Riminophenazines Phenoxazine and asymmetrical riminophenazine series}

Riminophenazinesexemplified by clofazimine, originally a dye shows potential to shorten treatment of multidrug-resistant tuberculosis, but skin coloration due to accumulation in fatty tissue and organs is a major side-effect. To reduce discoloration, TBI-166 was selected from 69 riminophenazine derivatives and first preclinical data suggests that it has retained the same antimycobacterial properties ${ }^{4851}$.

\section{Future prospective of the new advances of anti-TB drugs}

The mostimportant challenge in TB drug development is the difficulty in identifying new compounds with activity against $M$. tuberculosis. Regimens against TB should kill both the rapidly growing mycobacteria and the persisting mycobacteria in lesions [42]. New chemotherapeutic advances may arise from optimizing the use of existing anti-TB drugs, repurposing existing antibiotics for use as anti-TB drugs, or the discovery and development of new chemical entities. Among the drugs currently in clinical trials for TB are examples from each category (Table 2) [8]. Development of new medicines is a complex, lengthy, and expensive process, involving a series of stages (discovery, lead identification, and lead optimization), ultimately leading to preclinical development ${ }^{3940}$.

The emergence of MDR- and XDR-TB has spurred interest in the development of new drugs. Recently, the first report of phase 2 clinical trial of TMC207 for the treatment of MDR-TB has 
been published. Adding TMC207 to standard therapy for MDR-TB reduced the time to conversion to a negative sputum culture, as compared with placebo. TMC207, a first-in-class diarylquinoline, has a unique mechanism of action targeting the c subunit of adenosine triphosphate synthase and exhibits a long half-life. TMC207 plus rifapentine plus PZA gave once weekly was more active than the current standard regimen of RMP plus INH plus PZA given five times per week in murine TB, suggesting that it has the potential to be used to develop a fully intermittent once-weekly regimen.PA-824 is a novel nitroimidazole-oxazine, inhibits mycolic acid and protein biosynthesis and kills non-replicating MTB as well.PA-824 Enhances bactericidal activity of RMP and PZA in a murine model of TB. The combination of PA-824, moxifloxacin, and PZA cured mice more rapidly than the first-line regimen of RMP, INH and PZA in a murine model of TB, implying the potential to shorten the duration of TB treatment. Other imidazole-oxazole, diamine, and pyrrole compounds are being investigated in clinical phase 1 or 2 drug trials as well, yet none of the compounds will have passed phase 3 to be available before the year $2011^{77}$.

\section{Conclusions}

The emergence of MDR-TB and XDR-TB, the need for new TB drug regimens and rapid DST is intuiting globally. The prospect of new TB regimens is exciting because patients have had to rely on a single lengthy treatment option for decades. New DST assays to detect resistance can be developed before repurposed drugs come to market, and early in the implementation of new medicines. New drugs are needed that have strong, synergistic and complementary activities against various $\mathrm{M}$. tuberculosis subpopulations to shorten TB treatment, be effective against MDR-TB/XDR-TB, new combinations of new and old drugs to (or "intending to") avoiding misuse of the new compounds and prevent the emergence of different resistance. This calls for the development of a broader drug pipeline and a new paradigm for the identification of novel TB drug combinations.

The recent licensing of aquiline and the encouraging performance of sterilizing drugs such as the 8-methoxyfluoroquinolines and nitroimidazoles in Phase IIb clinical trials are concrete signs of progress towards shorter therapy. Long-term investment, political commitment, and scientific endeavor will be crucial to ensure that progress is sustained and the benefits of recent advances reach those in the greatest need. 


\section{Acknowledgements}

All the authors of the manuscript thank and acknowledge their respective Universities and Institutes.

\section{Conflict of Interest}

The authors declare that they have no conflict of interest.

\section{Authors' Contribution}

MHB, FM and IA collected literatures and drafted the paper. KD, and HMNI participated in the manuscript preparation, drafted the manuscript. Moreover, MHB, FM, IA, KD and HMNI reviewed and performed the final check. All the authors read and approved the final manuscript.

\section{Orcid}

\section{Mohammed Hussen Bule}

http:// orcid.org/0000-0002-1040-1583

\section{Kuldeep Dhama}

http://orcid.org/0000-0001-7469-4752

https://livedna.net/?dna=91.4710

\section{REFERENCES}

1. Saifullah B., Hussein MZB., Al Ali SHH, 2012. Controlled-release approaches towards the chemotherapy of tuberculosis. Int. J. Nanomedicine, 7:5451-63. https://doi.org/10.2147/IJN.S34996.

2. Mitnick CD., McGee B., Peloquin CA, 2009. Tuberculosis pharmacotherapy: strategies to optimize patient care. Expert Opin. Pharmacother, 10:381-401. https://doi.org/10.1517/14656560802694564.

3. World Health Organization Global tuberculosis report 2013. Available from: http://apps.who.int/iris/bitstream/10665/191102/1/9789241565059 [Accessed 22 March 2017]. 
4. Ahmad S., Mokaddas E, 2009. Recent advances in the diagnosis and treatment of multidrugresistant tuberculosis. Respir. Med., 103:1777-90.

https://doi.org/10.1016/j.rmed.2009.07.010.

5. MDR_TB_FactSheet. Available from: http://www.who.int/tb/challenges/mdr/MDR_TB_FactSheet.pdf [Accessed 15 March 2017].

6. Gillespie SH, 2002. Evolution of drug resistance in Mycobacterium tuberculosis: clinical and molecular perspective. Antimicrob. Agents Chemother., 46:267-74.

7. Chiang C-Y., Centis R., Migliori GB, 2010. Drug-resistant tuberculosis: past, present, future. Respirol. Carlton Vic., 15:413-32. https://doi.org/10.1111/j.1440-1843.2010.01738.x.

8. Nuermberger EL., Spigelman MK., Yew WW, 2010. Current development and future prospects in chemotherapy of tuberculosis. Respirology, 15:764-78. https://doi.org/10.1111/j.1440-1843.2010.01775.x.

9. van der Werf MJ., Langendam MW., Huitric E., Manissero D, 2012. Multidrug resistance after inappropriate tuberculosis treatment: a meta-analysis. Eur. Respir. J., 39:1511-9. https://doi.org/10.1183/09031936.00125711.

10. Udwadia ZF., Amale RA., Ajbani KK., Rodrigues C, 2012. Totally drug-resistant tuberculosis in India. Clin Infect Dis Off Publ Infect. Dis. Soc. Am., 54:579-81. https://doi.org/10.1093/cid/cir889.

11. Velayati AA., Masjedi MR., Farnia P., Tabarsi P., Ghanavi J., Ziazarifi AH., et al, 2009. Emergence of new forms of totally drug-resistant tuberculosis bacilli: super extensively drug-resistant tuberculosis or totally drug-resistant strains in iran. Chest., 136:420-5. https://doi.org/10.1378/chest.08-2427.

12. Guidelines for the programmatic management of drug-resistant tuberculosis 2011 update. Available from: http://apps.who.int/iris/bitstream/10665/44597/1/9789241501583_eng.pdf [Accessed 15 March 2017]. 
13. WHO Guidelines for the programmatic management of drug-resistant tuberculosis: Emergency update 2008. Available from:

http://apps.who.int/iris/bitstream/10665/43965/1/9789241547581_eng.pdf [Accessed 22 March 2017].

14. Van Deun A., Maug AKJ., Salim MAH., Das PK., Sarker MR., Daru P., et al, 2010. Short, highly effective, and inexpensive standardized treatment of multidrug-resistant tuberculosis. Am. J. Respir. Crit. Care. Med., 182:684-92. https://doi.org/10.1164/rccm.201001-0077OC.

15. Lienhardt C., Raviglione M., Spigelman M., Hafner R., Jaramillo E., Hoelscher M., et al, 2012. New drugs for the treatment of tuberculosis: needs, challenges, promise, and prospects for the future. J. Infect. Dis., 205: 241-249. https://doi.org/10.1093/infdis/jis034.

16. Sloan DJ., Davies GR., Khoo SH, 2013. Recent advances in tuberculosis: New drugs and treatment regimens. Curr. Respir. Med. Rev., 9:200-10.

17. Worthington RJ., Melander C, 2013. Combination Approaches to Combat Multi-Drug Resistant Bacteria. Trends Biotechnol., 31:177-84. https://doi.org/10.1016/j.tibtech.2012.12.006.

18. CDC. World TB Day - March 24, 2013 Morbidity and Mortality Weekly. Vol. 62 / No. 11. Available From: https://www.cdc.gov/mmwr/preview/mmwrhtml/mm6211a1.htm?s_cid=mm6211a1_e. [Accessed 15 March 2017].

19. Muwonge A., Malama S., Johansen TB., Kankya C., Biffa D., Ssengooba W., et al, 2013. Molecular Epidemiology, Drug Susceptibility and Economic Aspects of Tuberculosis in Mubende District, Uganda. PLOS ONE, 8:e64745. Available from: http://journals.plos.org/plosone/article?id=10.1371/journal.pone.0064745 [Accessed 2 March 2017]. 
20. Klinkenberg E., Assefa D., Rusen ID., Dlodlo RA., Shimeles E., Kebede B., et al, 2014. The Ethiopian initiative to build sustainable capacity for operational research: overview and lessons learned. Public Health Action, 4:2-7. https://doi.org/10.5588/pha.14.0051.

21. Maqbool F., Bahadar H., Abdollahi M, 2014. Exposure to Mercury from Dental Amalgams: A Threat to Society. Arch. Ind. Hyg. Toxicol., 65:339-340. https://doi.org/10.2478/100041254-65-2014-2543.

22. Federal Ministry of Health Ethiopia Tuberculosis, Leprosy and TB/HIV Prevention and Control Programme Manual. n.d. Available From: http://www.who.int/hiv/pub/guidelines/ethiopia_tb.pdf [Accessed 22 March 2017]

23. Orenstein EW., Basu S., Shah NS., Andrews JR., Friedland GH., Moll AP., et al, 2009. Treatment outcomes among patients with multidrug-resistant tuberculosis: systematic review and meta-analysis. Lancet Infect. Dis., 9:153-61. https://doi.org/10.1016/S14733099(09)70041-6.

24. Adhvaryu M., Vakharia, 2011. Drug-resistant tuberculosis: emerging treatment options. Clin. Pharmacol. Adv. Appl., Vol. 2013:51-67. https://doi.org/10.2147/CPAA.S11597.

25. Kirimuhuzya C, 2012. Multi-Drug/Extensively Drug Resistant Tuberculosis (Mdr/Xdr-Tb): Renewed Global Battle Against Tuberculosis? Pere-Joan Cardona (Ed.). INTECH Open Access Publisher, pp: 3-32

26. Rai S.P., 2013. Tackling Extensively Drug Resistant Tuberculosis (XDR-TB). Indian J. Tuberc., 60:67-70.

27. Plan to Combat Extensively Drug-Resistant Tuberculosis Recommendations of the Federal Tuberculosis Task Force. Available From: https://www.cdc.gov/mmwr/preview/mmwrhtml/rr5803a1.htm. [Accessed March 2017]. 
28. Prasad R, 2012. Multidrug and extensively drug-resistant tuberculosis management: Evidences and controversies. Lung India Off Organ Indian Chest. Soc., 29:154-9. https://doi.org/10.4103/0970-2113.95321.

29. Lee M., Lee J., Carroll MW., Choi H., Min S., Song T., et al, 2012. Linezolid for Treatment of Chronic Extensively Drug-Resistant Tuberculosis. N. Engl J. Med., 367:1508-18. https://doi.org/10.1056/NEJMoa1201964.

30. Maqbool F., Mostafalou S., Bahadar H., Abdollahi M, 2016. Review of endocrine disorders associated with environmental toxicants and possible involved mechanisms. Life Sci., 145:265-73. https://doi.org/10.1016/j.lfs.2015.10.022.

31. Smith T., Wolff K.A., Nguyen L., 2012. Molecular Biology of Drug Resistance in Mycobacterium tuberculosis. In: Pathogenesis of Mycobacterium tuberculosis and its Interaction with the Host Organism, Pieters J, McKinney JD, (Eds.). vol. 374. Berlin, Heidelberg: Springer Berlin Heidelberg, pp: 53-80.

32. Hmama Z., 2013. Management of Drug-Resistant TB. In: Tuberculosis - Current Issues in Diagnosis and Management. Mahboub B, (Ed.) InTech Open Access Publisher, pp: 4763.

33. Machado D., Couto I., Perdigão J., Rodrigues L., Portugal I., Baptista P., et al, 2012. Contribution of Efflux to the Emergence of Isoniazid and Multidrug Resistance in Mycobacterium tuberculosis. PLOS ONE., 7:e34538, https://doi.org/10.1371/journal.pone.0034538.

34. Müller B., Borrell S., Rose G., Gagneux S., 2013. The heterogeneous evolution of multidrugresistant Mycobacterium tuberculosis. Trends Genet. TIG., 29:160-9. https://doi.org/10.1016/j.tig.2012.11.005.

35. Grover GS., Takkar J., 2008. Recent Advances in Multi-Drug-Resistant Tuberculosis and RNTCP. Indian J. Community Med. Off Publ. Indian Assoc. Prev. Soc. Med., 33:219_ 23. https://doi.org/10.4103/0970-0218.43238. 
36. The Global Plan to Stop TB, 2011-2015. Geneva, World Health Organization, 2010. Available from: http://apps.who.int/iris/bitstream/10665/44437/1/9789241500340_eng.pdf [Accessed 15 March 2017].

37. Federal Ministry of Health Ethiopia 2009.Guideline for program and clinical management of drug resistant Tuberculosis. Addis Ababa. 2012. Available from: https://www.medbox.org/guidelines...clinical...programmatic-management...tb...ethio. [Accessed 15 March 2017].

38. Fiseha D., Kumssa H., Tefera M., Tesfaye A., Klinkenberg E., Yimer G, 2014. Ambulatory care for multidrug-resistant tuberculosis: lessons learned in Addis Ababa, Ethiopia. Public Health Action., 4:37-41. https://doi.org/10.5588/pha.14.0058.

39. Lienhardt C., Vernon A., Raviglione MC., 2010. New drugs and new regimens for the treatment of tuberculosis: review of the drug development pipeline and implications for national programmes. Curr. Opin. Pulm. Med., 16:186-93. https://doi.org/10.1097/MCP.0b013e328337580c.

40. Zumla AI., Gillespie SH., Hoelscher M., Philips PPJ., Cole ST., Abubakar I., et al, 2014. New antituberculosis drugs, regimens, and adjunct therapies: needs, advances, and future prospects. Lancet Infect. Dis., 14:327-40. https://doi.org/10.1016/S14733099(13)70328-1.

41. Spigelman MK, 2007. New Tuberculosis Therapeutics: A Growing Pipeline. J. Infect. Dis., 196:28-34. https://doi.org/10.1086/518663.

42. Alqahtani JM., Asaad AM., 2014. Anti-Tuberculous Drugs and Susceptibility Testing Methods: Current Knowledge and Future Challenges. Mycobact Dis., 4: 140. https://doi.org/10.4172/2161-1068.1000140.

43. Chang K-C., Yew W-W., 2013. Management of difficult multidrug-resistant tuberculosis and extensively drug-resistant tuberculosis: update 2012. Respirol Carlton Vic., 18:8-21. https://doi.org/10.1111/j.1440-1843.2012.02257.x. 
44. Cheepsattayakorn A., Cheepsattayakorn R., 2012. Novel Compounds and Drugs and Recent Patents in Treating Multidrug- Resistant and Extensively Drug-Resistant Tuberculosis. Recent Patents Anti-Infect. Drug Disc., 7:141-56. https://doi.org/10.2174/157489112801619683.

45. Matteelli A., Carvalho AC., Dooley KE., Kritski A., 2010. TMC207: the first compound of a new class of potent anti-tuberculosis drugs. Future Microbiol., 5:849-58. https://doi.org/10.2217/fmb.10.50.

46. Zhang T., Li S-Y., Williams KN., Andries K., Nuermberger EL., 2011. Short-Course Chemotherapy with TMC207 and Rifapentine in a Murine Model of Latent Tuberculosis Infection. Am. J. Respir. Crit. Care Med., 184:732-7. https://doi.org/10.1164/rccm.201103-0397OC.

47. Goel D., 2014. Bedaquiline: A novel drug to combat multiple drug-resistant tuberculosis. J. Pharmacol. Pharmacother., 5:76-8. https://doi.org/10.4103/0976-500X.124435.

48. Sacksteder KA., Protopopova M., Barry CE., Andries K., Nacy CA., 2012. Discovery and development of SQ109: a new antitubercular drug with a novel mechanism of action. Future Microbiol., 7:823-37. https://doi.org/10.2217/fmb.12.56. 
Table 1 Structures of first-line antituberculosis drugs.

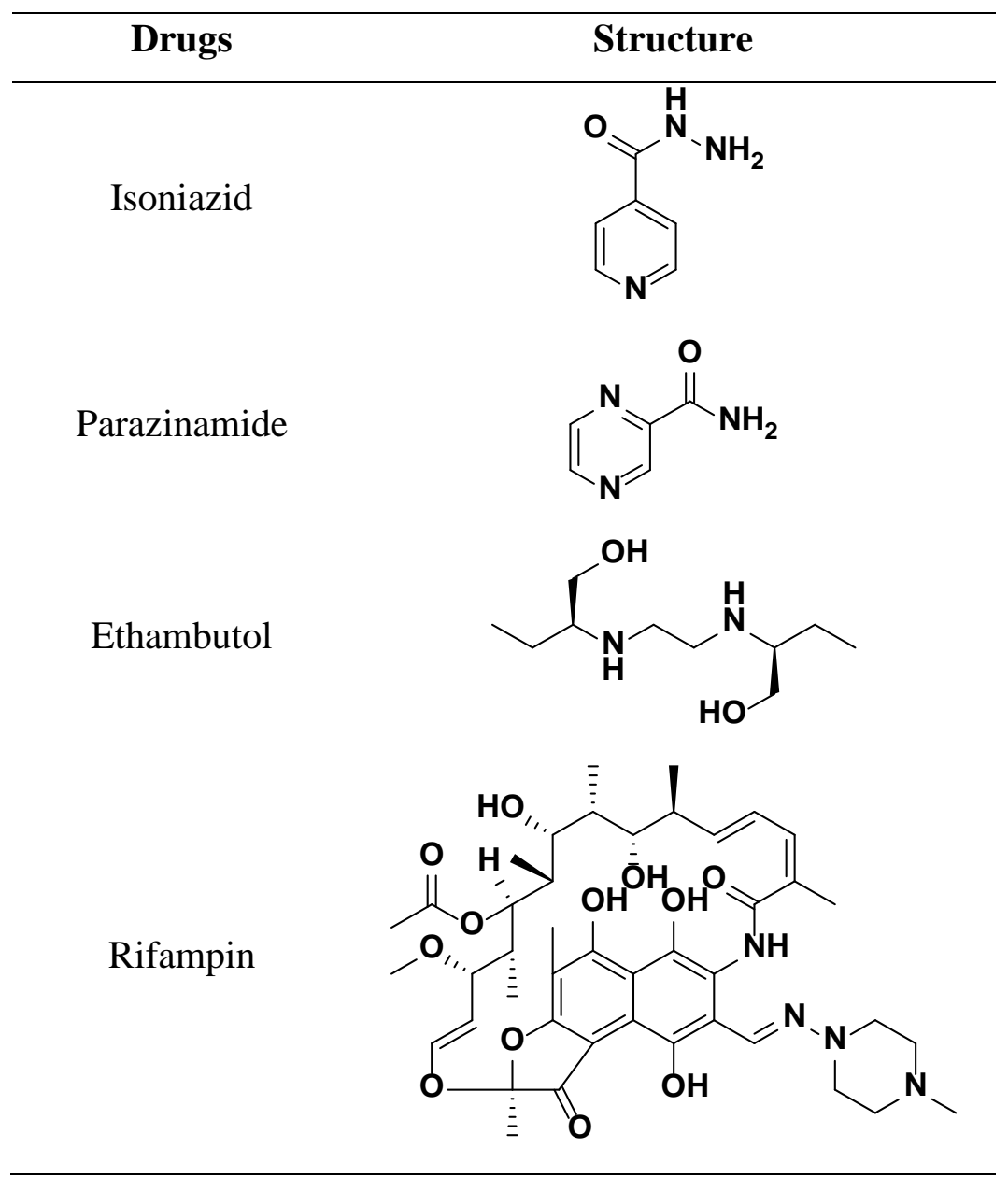


Table 2 New Anti- TB drug candidates in clinical development.

\begin{tabular}{|c|c|c|c|}
\hline Drug & Class & Mechanism (s) of action & Target \\
\hline Rifapentine & Rifamycine & Inhibition of RNA synthesis & $\begin{array}{l}\text { DNA-dependent } \\
\text { RNA polymerase }\end{array}$ \\
\hline Moxifloxacin & Fluoroquinolone & Inhibition of DNA synthesis & DNA gyrase \\
\hline Gatifloxacin & Fluoroquinolone & Inhibition of DNA synthesis & DNA gyrase \\
\hline TMC207 & Diarylquinoline & Inhibition of ATP synthesis & $\begin{array}{c}\text { F1F0 proton ATP } \\
\text { synthase }\end{array}$ \\
\hline PA-824 & Nitroimidazo-oxazine & $\begin{array}{l}\text { Inhibition of cell wall lipid synthesis, } \\
\text { inhibition of protein synthesis, } \\
\text { indirect effects of nitric oxide } \\
\text { generation (?) }\end{array}$ & Unknown \\
\hline OPC-67683 & Nitroimidazo-oxazole & $\begin{array}{l}\text { Inhibition of cell wall lipid synthesis, } \\
\text { inhibition of protein synthesis, } \\
\text { indirect effects of nitric oxide } \\
\text { generation (?) }\end{array}$ & Unknown \\
\hline SQ109 & Diethylamine & Inhibition of cell wall synthesis & Unknown \\
\hline Linezolid & Oxazolidinone & Inhibition of protein synthesis & $\begin{array}{l}\text { Ribosomal } \\
\text { initiation complex }\end{array}$ \\
\hline PNU-100480 & Oxazolidinone & Inhibition of protein synthesis & $\begin{array}{c}\text { Ribosomal } \\
\text { initiation complex }\end{array}$ \\
\hline AZD5847 & Oxazolidinone & Inhibition of protein synthesis & $\begin{array}{c}\text { Ribosomal } \\
\text { initiation complex }\end{array}$ \\
\hline LL-3858 & Pyrrole & Unkown & Unkown \\
\hline
\end{tabular}




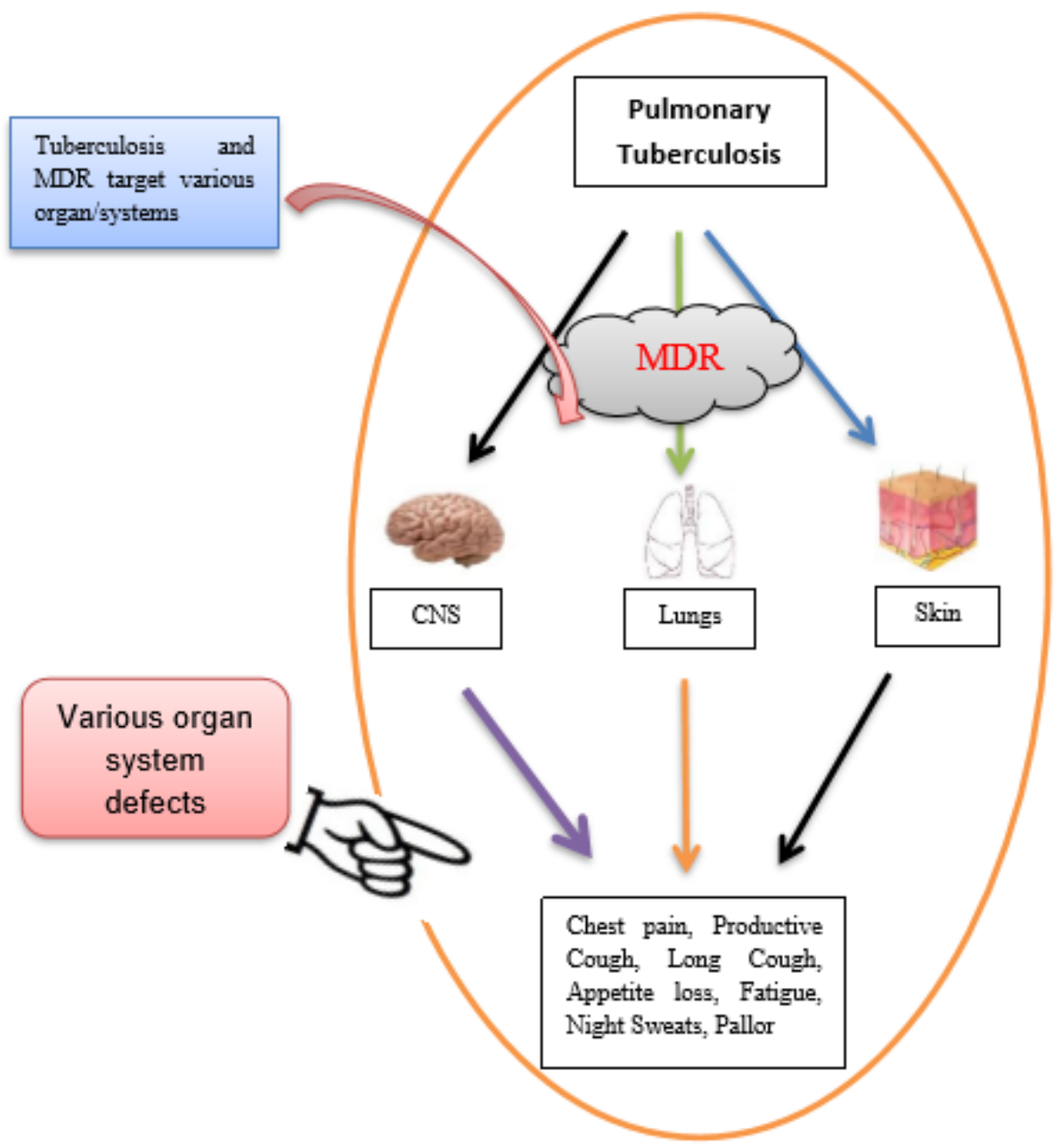

Figure 1 Organs/System effects the pulmonary tuberculosis and mechanism involved in MDR. 

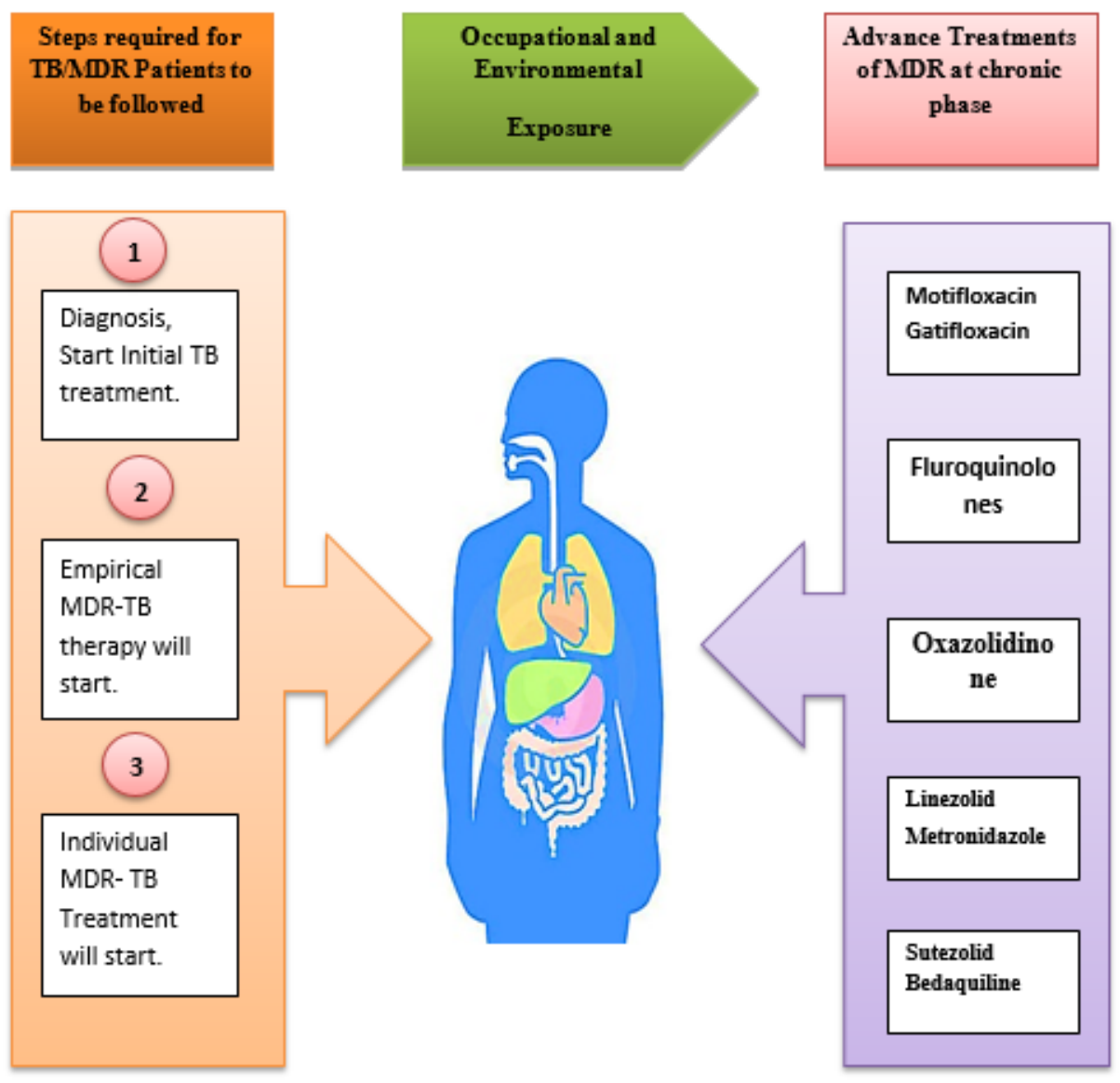

Figure 2 Different stages of MDR with different therapeutic strategies. 


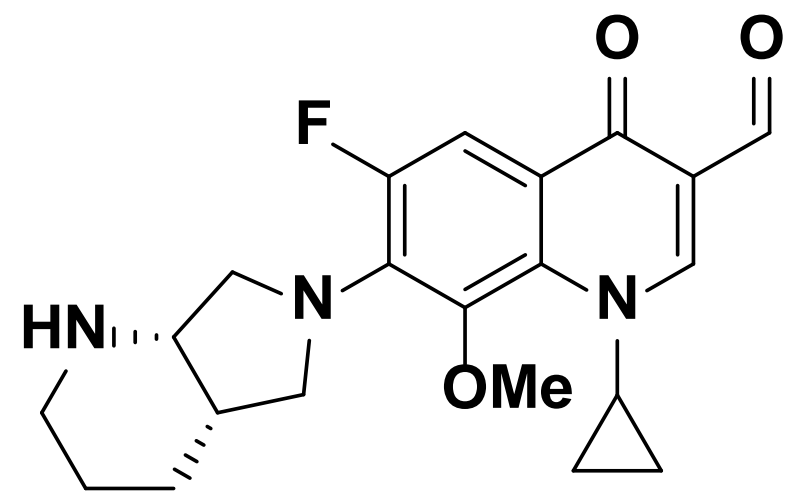

Figure 3 Chemical structure of Moxifloxacin.

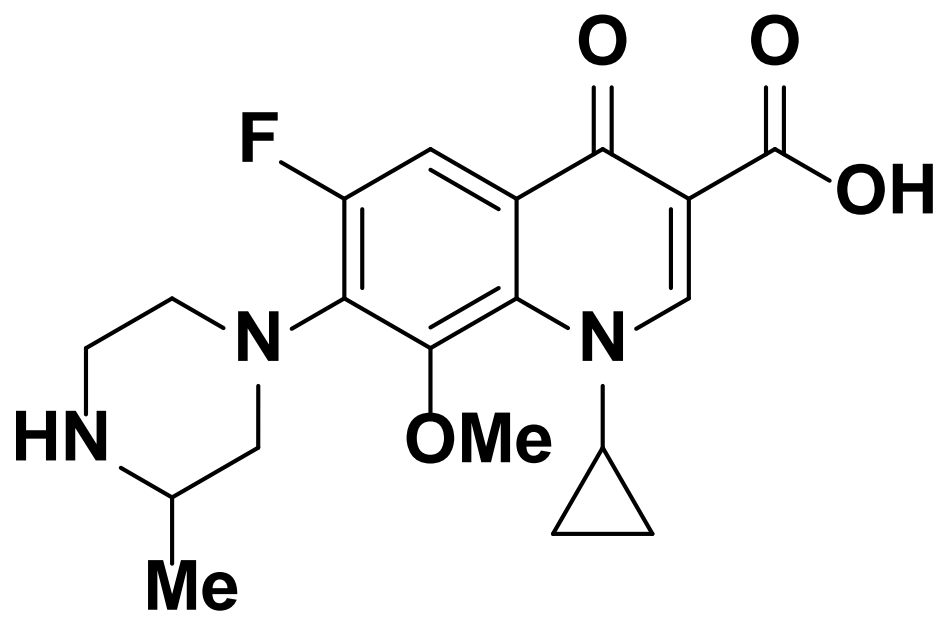

Figure 4 Chemical structure of Gatifloxacin.

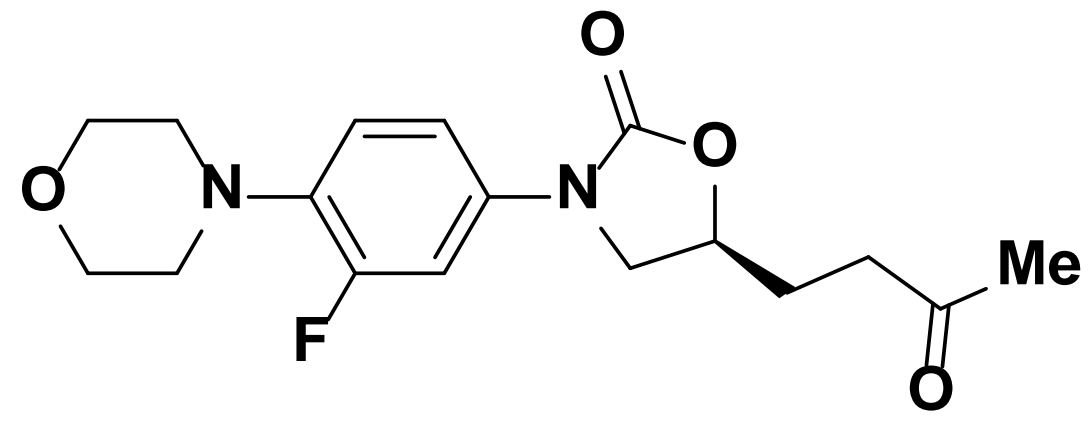

Figure 5 Chemical structure of Linezolid. 


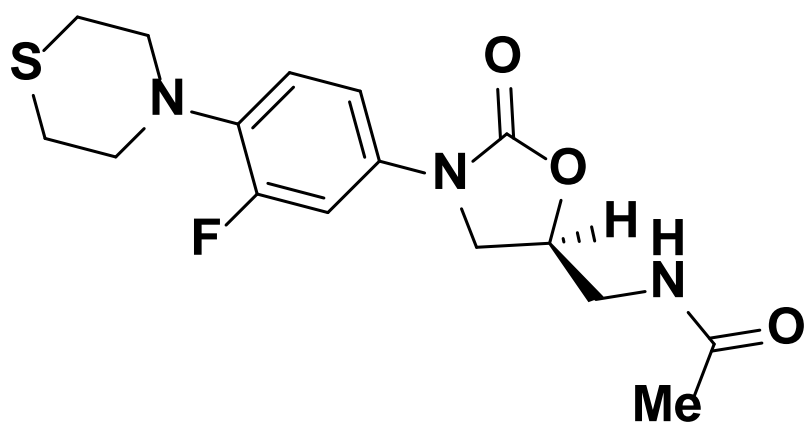

Figure 6 Chemical structure of PNU 100480.

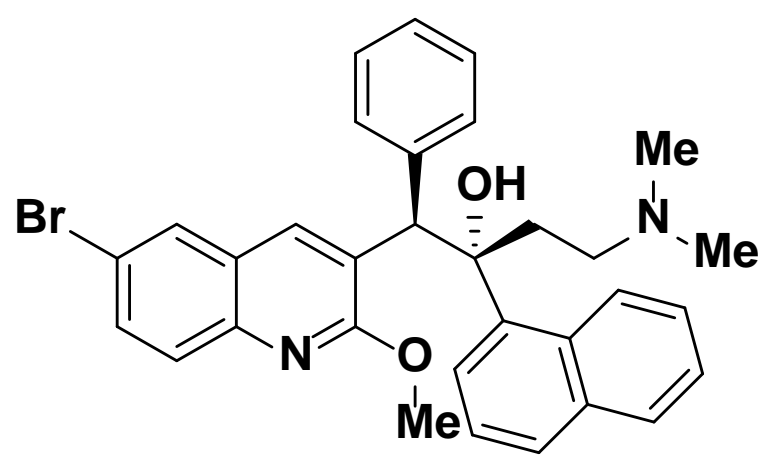

Figure 7 Chemical structure of TMC-207 (Bedaquiline).

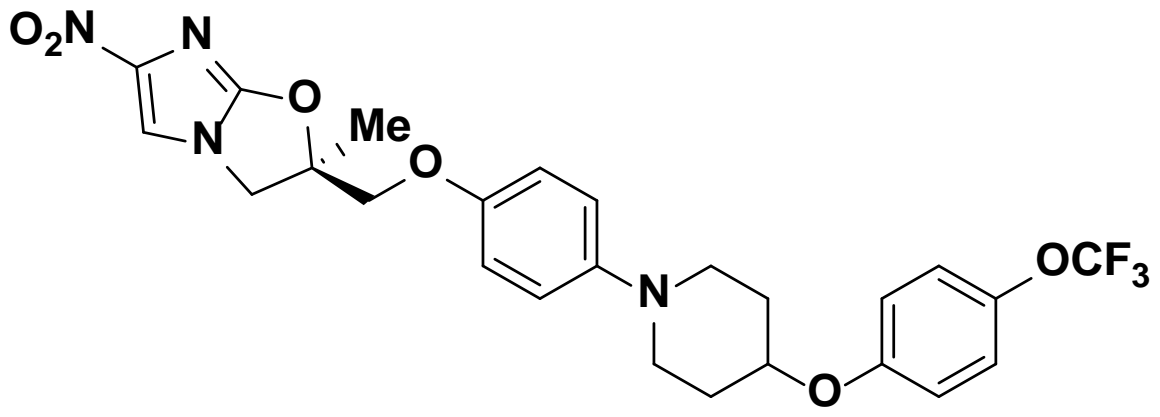

Figure 8 Chemical structure of OPC-67683. 


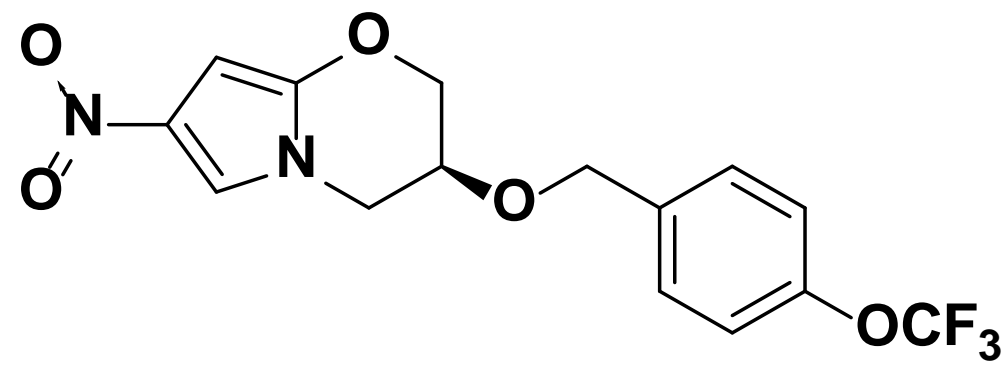

Figure 9 Chemical structure of PA-824.

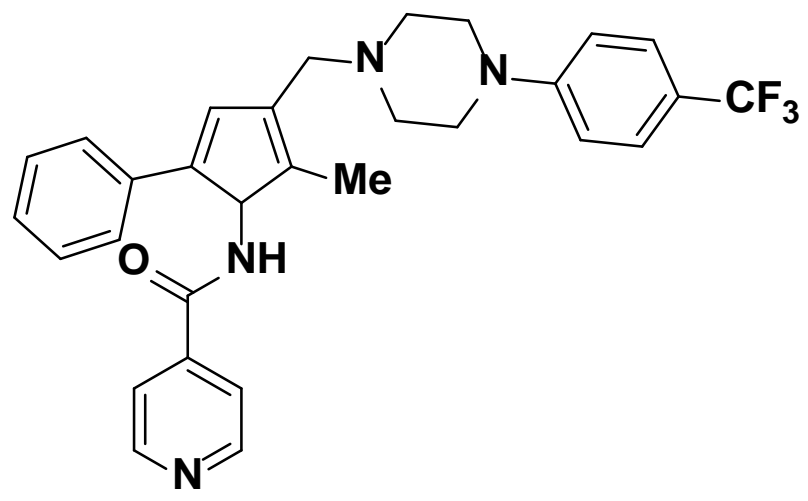

Figure 10 Chemical structure of Pyrrole (LL3858).

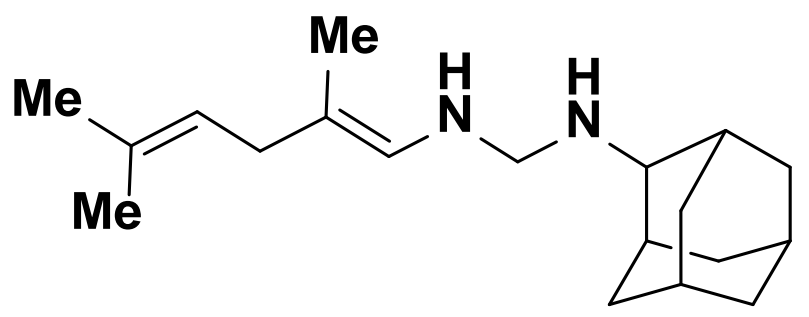

Figure 11 Chemical structure of SQ109. 


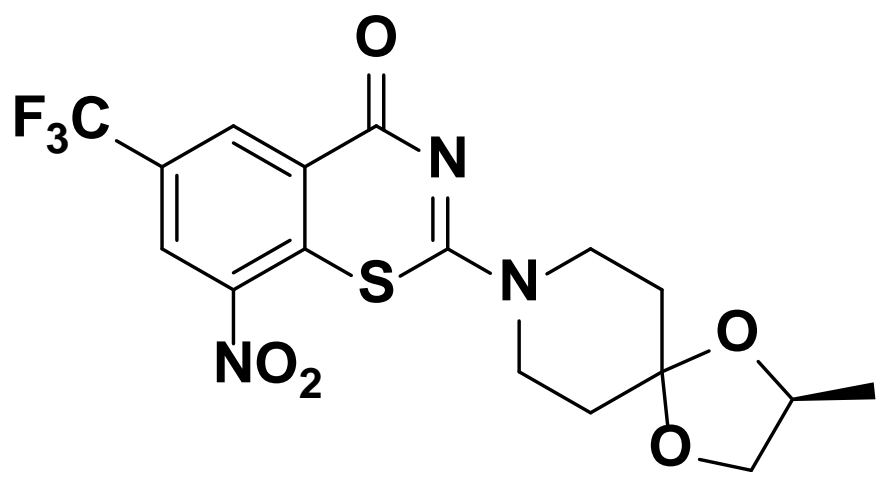

Figure 12 Chemical structure of Benzothiozinones. 